\title{
Global existence and nonexistence of strong solutions to pseudo-parabolic equations with variable exponents
}

\author{
Le Nhan ${ }^{1}$ and Truong Le ${ }^{2}$ \\ ${ }^{1}$ Ho Chi Minh City University of Technology and Education \\ ${ }^{2}$ University of Economics Ho Chinh Minh City
}

April 28, 2020

\begin{abstract}
This paper deals with a pseudo-parabolic equation involving variable exponents under Dirichlet boundary value condition. The authors first develop the potential well method to prove a threshold result on the existence and nonexistence of global solutions to the equations when initial energy is less than the mountain pass level $\$ \mathrm{~d} \$$. By borrowing the idea from (missing citation); (missing citation) we also show some existence and nonexistence results with high energy initial data. In this case a new characterization for nonexistence of solution is given. These results extend and improve a recent result obtained by Di et al. (2017) (missing citation) and Liao (2019) (missing citation); (missing citation).
\end{abstract}

\section{Hosted file}

NhTruong-Mar-31-20.pdf available at https://authorea.com/users/308981/articles/439996-globalexistence-and-nonexistence-of-strong-solutions-to-pseudo-parabolic-equations-with-variableexponents

\section{References}

